\title{
Textile Fabric Defect Detection
}

\author{
Sneha Kulkarni ${ }^{1}$, Kajal Jojare ${ }^{2}$, Vaishnavi Bhosale ${ }^{3}$, Priyanka Arude ${ }^{4}$ \\ Professor, E\&TC Department, NBN Sinhgad School of Engineering, Pune. \\ Student, E\&TC Department, NBN Sinhgad School of Engineering, Pune.
}

\begin{abstract}
The main objective of our project is detecting the fault of fabric. In textile industry automatic fabric inspection is important to maintain the quality of fabric. In our project horizontal, Vertical and oil spot such defects are detected using neural network tool. These systems are introducing defect detection using histogram and Neural Network Tool for the inspection textured material. The system acquires fabric images by image acquisition device \& converts that image into binary image by restoration and thresholding techniques. ARM 7 is used for processing all actions. This system is based on MATLAB 8.0.0.873.
\end{abstract}

Keywords: GSM, Histogram, Threshold, DWT.

\section{INTRODUCTION}

The Indian textile production has a major impact on the world economy through millenniums. At present fabric inspection depends on human sight, the result of inspection influenced by the physical and mental condition of inspector. Now, all the textile industries aim to produce good quality fabrics with high production rate. In the textile sector, there are huge losses due to faulty fabrics. The fabric is obtained by interweaving of warp and weft yarn. The faults found in the fabrics are around $80-85 \%$ of the defects in the garment industry. These faults are obtained in the fabrics due to irregular weaving of warp and weft yarn in the weaving process. Some of these fabric defects are visible, while others are not. Again some fabric defects may be identified during weaving and some after weaving process. The manual inspection of fabric material is not economical and work is very dull. Hence, the investment in automated fabric defect detection is economic when reduction in labour cost and other benefits are considered. Detection of fabric defects can be considered as a texture segmentation and identification problem, since textile faults normally have textural features that are different from features of the original fabric. The high production speed and the large flexibility required by customers' urge to automated defect detection of the quality assurance system. In the traditional textile industry, this task is carried out by human visual inspection. Manual inspection is usually a difficult task due to the small scale of detects and the large scale of inspected surface. In the case of the weaving sector, inspection is performed at the end of the manufacturing stage. Large batches of fabric rolls are manually inspected and actions are performed off-line of the production system. Employing computer vision automation directly on the production stage will improve the on-line reaction of the manufacturing staff and reduce the number of defects. Besides the high processing speed, computer vision systems can offer robust detection and large flexibility. Automation based on image processing does not suffer of human limitations and could entirely replace traditional methods. Automated visual inspection relies on material properties as texture. Texture analysis techniques for fabric defect detection allows determining texture features and statistically segment defects.

\section{Methodology}

The overview of the system as shown in this figure gives the detailed description of each block is explained below:

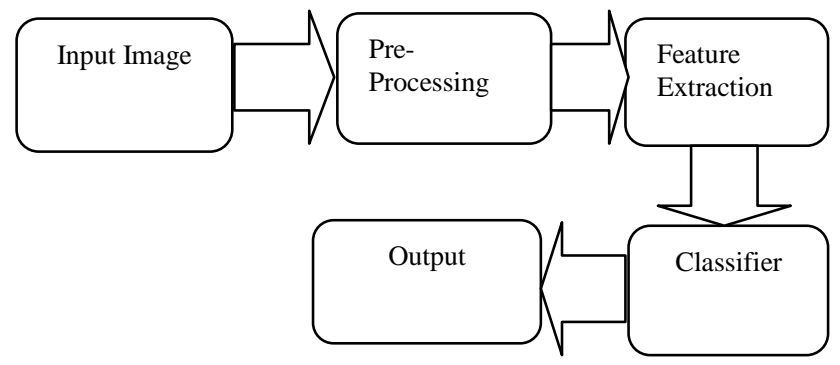

Fig. Block Diagram

\section{A. Input Image}

In this system the image is taken from a database. The database is collection of different faulty images and it will also include no-fault images.

\section{B. Pre-Processing}

\section{Histogram}

Histogram provides a global description of the appearance of an image. Histogram that acts as a graphical representation of total distribution in image. It plots the number of pixels in an image at each different intensity value of that image.

2. Thresholding

In the threshoding a gray level image is converted into 
bi-level image using an optimal threshold. There are two types of Thresholding:

\section{1) Global Thresholding}

2) Local or Adaptive Thresholding.

The role of thresholding is to extract the pixels from some image which represents an object. Though the information is binary the pixels represents range of intensities.

\section{Noise Removal}

The unwanted pixel is removed by using filters. Here we can use WIENER filter to reduce the noise. The WIENER filter can be used to restore images in the presence of blur as well as noise.

\section{Feature Extraction}

If the input data to an algorithm is too large to be processed and for an simplification of getting output then it can be transformed into reduced set of feature. There are many techniques for feature extraction. In feature extraction various features like shape, size etc. are extracted through programming.

\section{Classifier}

The basic function of the classifier to compare the feature vectors obtained from the feature extractor and arrived at a decision to classify the object within the image correctly.

\section{ALgORITHM}

1. Start.

2. Read an image of fabric.

3. Apply discrete wavelet transform.

4. Do image segmentation for separate defect from image.

5. Noise removal from segmented image.

6. Apply feature extraction.

7. Classification of defect using Neural Network.

8. Send the result to controller.

9. When key press all the result will be send to the engineer via GSM modem.

10. End.

\section{IV.SYSTEM ARCHITECTURE}

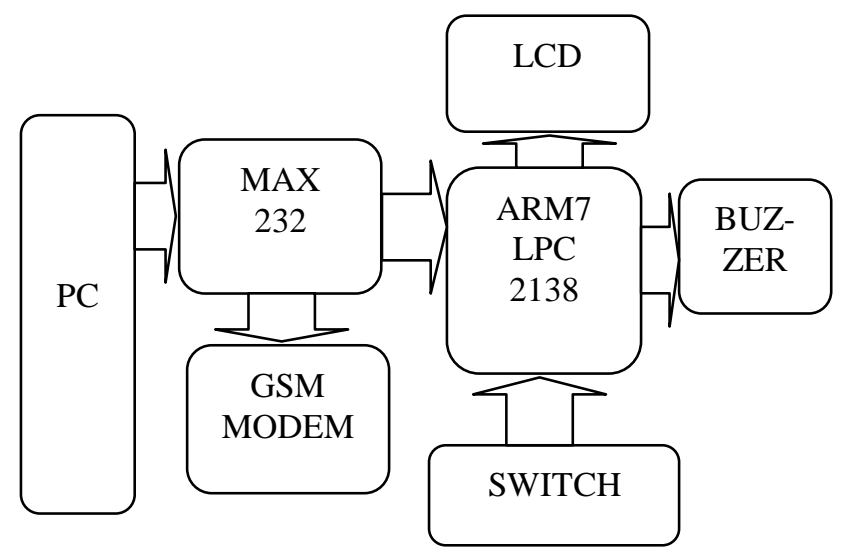

Fig. System Architecture.
For fabric fault detection system gives the images of fabric from database to the controller for processing all action. The count is incremented accordingly after detection of fabric i.e. whether that fabric is faulty or non-faulty. GSM for message sending and buzzer for indication after the completion of analysis all the fabric then when user will press the switch daily report of the fabric analysis will be send to admin or production engineer.

This system use the neural network technique for analysis of the fabric fault we will extract the feature from the fault fabric give it to the neural network and when we pass the test image to the algorithm with help of neural network we well be able to analyse the fault and all the details of the fault in the fabric is stored in the database.

\section{V.RESULT}

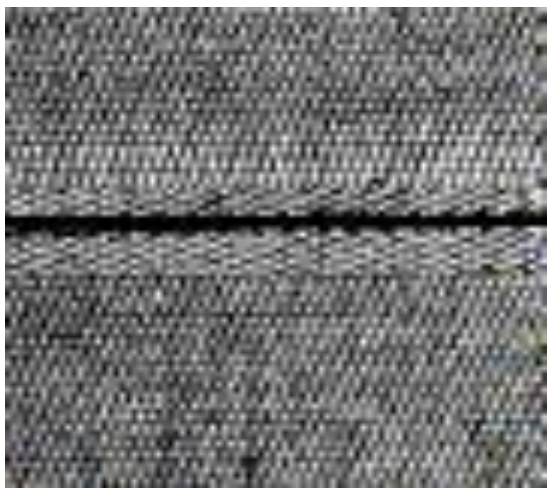

Fig. Input Image.

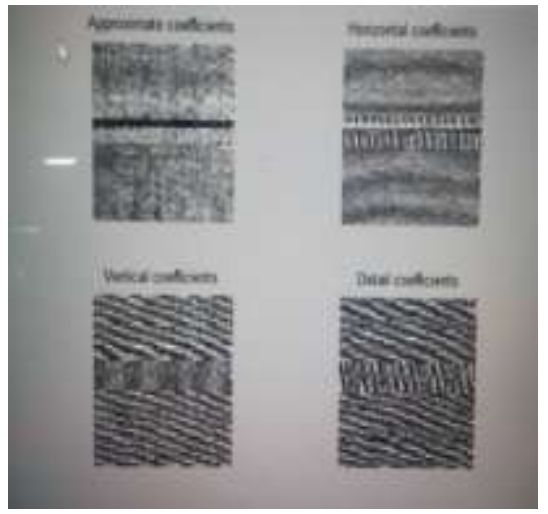

Fig. DWT Result.

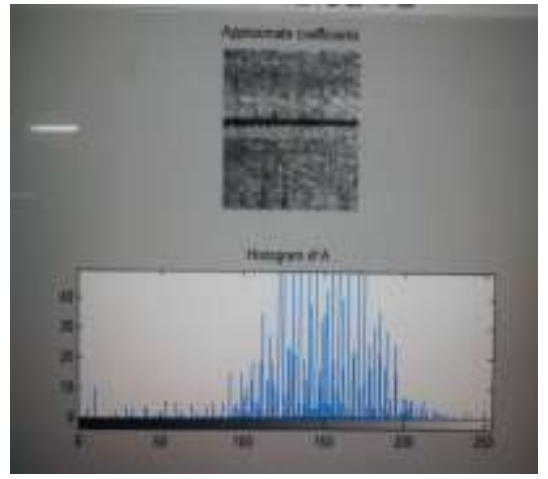

Fig. Histogram Output 


\section{CONCLUSION}

The MATLAB implementation is done for fault identification such as oil spot, horizontal, vertical on fabric can be detected. The manual inspection is very tedious and time consuming hence by using automatic visual inspection reduces the man power and improved the quality of fabric by using histogram and thresholding technique.

\section{REFERENCES}

1. Prof.M.S.Biradar, Prof.Sharda P.Jadhav "Fabric defect detection using neural network technique" IOSR Journal of Electronics and Communication Engineering,vol.4 pp 70-76 Mar-Apr.2014.

2. R.C.Gonzalez, R.E.Woods, S.L.Eddins, "Digital Image Processing using Matlab”, 2005. pp. 76-104, pp.142-166

3. Jagrti Patel, Meghna Jain, Papiya Dutta "Detection of Faults Using Image Processing Technique", Asian Journal of Engineering and Applied Technology, Vol.2 pp.36-39 May-2013

4. Tamnun F Mursalin, Fajrana Zebin Eishita, Ahmed Ridwanul Islam "Fabric Defect Inspection System Using Neural Network And Microcontroller" Journal of Theortical and Applied Information Technology,

5. Rakhil k.S., Asha s. "Improved fabric defect detection and pattern classification using random transform",An ISO 3297:2007 (Certified Organizations)Vol-4,Issue),July 2015.

6. Navneet Kaur, Mandeep Dalal "Application of machine vision technique in textile (Fabric)quality Analysis" IOSR Journal of Engineering, Apr.2012,Vol. 2(4)

7. Vikrant Tiwari, Gaurav Sharma "Automatic Fabric Fault Detection Using Morphological Operations Bit Plane” IJCSNS International Journal of Computer Science and Network Security, VOL.15 No.10, October 2015.

8. Jagruti Mahure, Y.C.Kulkarni "Fabrics Fault Processing Using Image Processing Technique in MATLAB" IJST Vol.4, Issue 2, April-June 2013.

9. Sujit Ahirrao, Swapnil Bahulekar, Balkrushna Chaudhary "Fabric defect detection using Fourier analysis” IJEST, 25 March 2015.

10. Xie Xianghua "A Review of recent advances in surface defect detection using texture analysis technique" Electronic Letters on Computer Vision and Image Analysis, 18 April 2008. 